\title{
Article \\ The Association between Oral Health-Related Quality of Life, Loneliness, Perceived and Objective Social Isolation-Results of a Nationally Representative Survey
}

\author{
André Hajek *(i) and Hans-Helmut König (D) \\ Hamburg Center for Health Economics, Department of Health Economics and Health Services Research, \\ University Medical Center Hamburg-Eppendorf, 20246 Hamburg, Germany; h.koenig@uke.de \\ * Correspondence: a.hajek@uke.de; Tel.: +49-40-7410-52877
}

Citation: Hajek, A.; König, H.-H. The Association between Oral Health-Related Quality of Life, Loneliness, Perceived and Objective Social Isolation-Results of a Nationally Representative Survey. Int J. Environ. Res. Public Health 2021, 18, 12886. https://doi.org/10.3390/ ijerph182412886

Academic Editors:

Kelvin Afrashtehfar and Paul B. Tchounwou

Received: 8 November 2021 Accepted: 30 November 2021 Published: 7 December 2021

Publisher's Note: MDPI stays neutral with regard to jurisdictional claims in published maps and institutional affiliations.

Copyright: (c) 2021 by the authors. Licensee MDPI, Basel, Switzerland. This article is an open access article distributed under the terms and conditions of the Creative Commons Attribution (CC BY) license (https:// creativecommons.org/licenses/by/ $4.0 /)$.

\begin{abstract}
The aim was to examine the association between oral health-related quality of life and loneliness and perceived as well as objective social isolation. Data were used from a nationally representative survey with $n=3075$ (late Summer 2021). The established Oral Health Impact Profile (OHIP-G5) was used to quantify oral health-related quality of life. Moreover, established tools were used to quantify the outcome measures (De Jong Gierveld loneliness scale, Bude/Lantermann scale and Lubben Social Network Scale). It was adjusted for several covariates in regression analysis. Linear regressions showed that low oral health-related quality of life is associated with higher loneliness $(\mathrm{B}=0.03, p<0.001)$, higher perceived social isolation $(\mathrm{B}=0.06, p<0.001)$ and higher objective social isolation ( $\mathrm{B}=0.07, p<0.05$ ). Further regressions were performed (e.g., stratified by denture usage). Our study stressed the importance of low oral health-related quality of life for loneliness and social isolation (both perceived and objective). This knowledge is important to address individuals at risk. Future studies should clarify the underlying mechanisms.
\end{abstract}

Keywords: oral health; oral health-related quality of life; loneliness; social isolation; social exclusion

\section{Introduction}

Loneliness, perceived social isolation and objective social isolation are gaining increasing awareness in the focus of research and the public-particularly in times of the COVID-19 pandemic, where social distancing is a main challenge. These aforementioned social needs are associated with subsequent morbidity and mortality $[1,2]$ - which underlines the importance of these factors.

Various studies have identified the determinants of loneliness, perceived social isolation and objective social isolation [1,2]. Such determinants include sociodemographic factors, lifestyle-related, psychological and health-related factors [3-6].

However, thus far, there is limited knowledge regarding the association between oral health-related quality of life and these social needs.

Most of the existing studies in this area focused on the association between objective oral health (e.g., decayed teeth or dental caries) [7-13] rather than (perceived) oral healthrelated quality of life $[14,15]$ and social needs (in terms of loneliness, perceived social isolation and objective social isolation). Most of the studies found an association between oral health and loneliness. However, existing studies often used data from small samples in quite specific regions and specific age groups (e.g., children or individuals in late life). In sum, there is a lack of studies analyzing the association between oral health-related quality of life and loneliness, perceived social isolation and objective social isolation based on data from the general adult population using nationally representative samples. Our aim was, therefore, to examine the association between oral health-related quality of life and loneliness, perceived social isolation as well as objective social isolation. This in turn is 
important to identify individuals at risk for high loneliness, perceived social isolation and objective social isolation.

The three social needs refer to different concepts. The outcomes are somewhat correlated (please see the Results section), but did not measure the same phenomenon. While loneliness refers to the discrepancy between actual and desired social relations [16], perceived social isolation refers to emotions of being left out from society [17] and objective social isolation refers to infrequent social contacts or rare social activities [18]. For further details, please see [19].

\section{Materials and Methods}

\subsection{Sample}

For our study, data were derived from a nationally representative online survey covering individuals aged 18 to 70 years. All participants lived in Germany. The sample size equaled 3075 individuals. It was performed from late August (24th) to early September (3rd). A well-established market research company recruited the participants. An online sample was used in a way that it matches the distribution of gender, age and federal state in the German population [20].

\subsection{Outcomes}

Loneliness was quantified using the De Jong Gierveld Loneliness scale (six-item version) [21]. Three items were recoded. The score was calculated by averaging the items. The score ranges from 1 to 4 , with higher values indicating higher loneliness. In our study, Cronbach's alpha was 0.78. It has been shown that this widely used tool (e.g., [22-24]) has good to very good psychometric characteristics [21,25].

The Bude and Lantermann tool (with four items) was used to measure perceived social isolation [17]. All items were recoded. A score was computed by averaging the items (from 1 to 4 , higher values correspond to higher perceived social isolation). In our study, Cronbach's alpha was 0.92 .

Moreover, the six-item version of the Lubben Social Network Scale [26] was used to quantify objective social isolation. It should be highlighted that all items were recoded prior to computing a sum score for reasons of consistency. Consequently, the resulting sum score ranges from 0 to 30, with higher values reflecting higher objective social isolation. In our study, Cronbach's alpha was 0.87 . This scale has favorable psychometric characteristics [26].

\subsection{Independent Variables}

The established Oral Health Impact Profile (OHIP-G5) was used to assess oral healthrelated quality of life. It covers the four dimensions: (i) oral function, (ii) orofacial pain, (iii) appearance and (iv) psychosocial impact. It has good psychometric properties [27]. The OHIP-G5 ranges from 0 to 20, with higher scores indicating lower oral health-related quality of life. In our study, Cronbach's alpha was 0.85. A further item refers to denture usage (options: 1. without dentures, 2. with removable dentures; 3 . with complete dentures). Associations between oral health-related quality of life and the outcome measures can, thus, be stratified by denture usage, since it already proved to be a key predictor in the longer OHIP-G-versions [28,29].

It was adjusted for covariates as follows: sex, age, presence of children under 18 years in the same household (no; yes), marital status (married, living together with spouse; married, not living together with spouse; single; widowed; divorced), highest educational degree (upper secondary school; qualification for applied upper secondary school; polytechnic Secondary School; intermediate Secondary School; Lower Secondary School; currently in school training/education; without school-leaving qualification) and employment status (full-time employed; retired; other). Moreover, it was adjusted for smoking status (yes, daily; yes, sometimes; no, not anymore; never smoker), alcohol intake (daily; several times per week; once a week; 1-3 times per month; less often; never) and sports activities (no sports activity; less than one hour a week; regularly, $1-2 \mathrm{~h}$ a week; regularly, $2-4 \mathrm{~h}$ a week; 
regularly, more than $4 \mathrm{~h}$ a week). Furthermore, vaccination against COVID-19 (no; yes), self-rated health (from $1=$ very bad to $5=$ very good) and chronic diseases (absence of chronic diseases; presence of at least one chronic disease) were used as covariates.

\subsection{Statistical Analysis}

First, sample characteristics are shown stratified by oral health-related quality of life (dichotomized, first category: score of zero; second category: scores of one or higher). Thereafter, bivariate correlations are displayed (additionally stratified by denture usage). Subsequently, multiple linear regressions were performed with oral health-related quality of life as key independent variable (first regression: with loneliness as outcome measure; second regression: with perceived social isolation as outcome measure; third regression: with objective social isolation as outcome measure). Further regressions were calculated and stratified by denture usage. The level of significance was set at $p<0.05$. Stata 16.1 (Stata Corp., College Station, TX, USA) was used for performing statistical analyses.

\section{Results}

\subsection{Sample Characteristics}

In our total sample, the mean age equaled 44.5 years, SD: 14.8 years. It ranged from 18 years to 70 years and $51.1 \%$ were female.

The average OHIP-G5 score was 2.2 (SD: 3.3), ranging from 0 to 20. Stratified by denture usage, the average OHIP-G5 score was 1.9 (SD: 3.0, 0 to 20) among individuals without dentures, 4.0 (SD: 4.5, 0 to 17) among individuals with removable dentures and 3.3 (SD: $3.8,0$ to 16) among individuals with complete dentures.

Stratified by oral health-related quality of life, sample characteristics are further displayed in Table 1. Dichotomized oral health-related quality of life was associated with most of the other variables (except for age, presence of children under 18 years in the same household, alcohol intake and vaccination against COVID-19).

Table 1. Sample characteristics stratified by oral health-related quality of life (first category: score of zero; second category: scores of one or higher).

\begin{tabular}{|c|c|c|c|c|}
\hline Variables & & $\begin{array}{c}\text { Oral Health-Related } \\
\text { Quality of Life: Scores of } \\
\text { Zero } \\
(\mathbf{N}=1537)\end{array}$ & $\begin{array}{c}\text { Oral Health-Related } \\
\text { Quality of Life: Scores of } \\
\text { One or Higher } \\
(\mathbf{N}=\mathbf{1 5 3 8})\end{array}$ & \\
\hline & & & & $p$-value \\
\hline \multirow[t]{4}{*}{ Sex: n (\%) } & & & & 0.07 \\
\hline & Men & $782(52.1)$ & $720(47.9)$ & \\
\hline & Women & $754(48.0)$ & $816(52.0)$ & \\
\hline & Diverse & 1 (33.3) & $2(66.7)$ & \\
\hline $\begin{array}{c}\text { Age: mean } \\
\text { (standard deviation) }\end{array}$ & & $44.3(14.6)$ & $44.8(15.0)$ & 0.41 \\
\hline \multirow[t]{3}{*}{ Marital status: n (\%) } & & & & 0.03 \\
\hline & $\begin{array}{l}\text { Single/Divorced/Widowed/ } \\
\text { Married, not living } \\
\text { together with spouse }\end{array}$ & $627(47.8)$ & $686(52.2)$ & \\
\hline & $\begin{array}{l}\text { Married, living together } \\
\text { with spouse }\end{array}$ & 910 (51.6) & $852(48.4)$ & \\
\hline $\begin{array}{l}\text { Highest educational } \\
\text { degree: } \mathrm{n}(\%)\end{array}$ & & & & 0.01 \\
\hline
\end{tabular}


Table 1. Cont.

\begin{tabular}{|c|c|c|c|c|}
\hline Variables & & $\begin{array}{c}\text { Oral Health-Related } \\
\text { Quality of Life: Scores of } \\
\text { Zero } \\
(\mathbf{N}=1537)\end{array}$ & $\begin{array}{c}\text { Oral Health-Related } \\
\text { Quality of Life: Scores of } \\
\text { One or Higher } \\
(\mathbf{N}=\mathbf{1 5 3 8})\end{array}$ & \\
\hline & upper secondary school & $706(53.2)$ & $620(46.8)$ & \\
\hline & $\begin{array}{l}\text { qualification for applied } \\
\text { upper secondary school }\end{array}$ & $161(49.1)$ & $167(50.9)$ & \\
\hline & $\begin{array}{l}\text { polytechnic Secondary } \\
\text { School }\end{array}$ & $78(46.4)$ & $90(53.6)$ & \\
\hline & $\begin{array}{l}\text { intermediate Secondary } \\
\text { School }\end{array}$ & $431(48.5)$ & $457(51.5)$ & \\
\hline & Lower Secondary School & $157(45.2)$ & $190(54.8)$ & \\
\hline & $\begin{array}{l}\text { currently in school } \\
\text { training/education }\end{array}$ & 1 (11.1) & $8(88.9)$ & \\
\hline & $\begin{array}{c}\text { without school-leaving } \\
\text { qualification }\end{array}$ & $3(33.3)$ & $6(66.7)$ & \\
\hline \multirow[t]{3}{*}{$\begin{array}{l}\text { Children under } 18 \\
\text { years in own } \\
\text { household: } \mathrm{n}(\%)\end{array}$} & & & & 0.67 \\
\hline & No & $1108(50.2)$ & $1098(49.8)$ & \\
\hline & Yes & $429(49.4)$ & $440(50.6)$ & \\
\hline \multicolumn{5}{|l|}{ Migration: n (\%) } \\
\hline & No migration background & $1395(51.2)$ & $1329(48.8)$ & \\
\hline & Migration background & $142(40.5)$ & $209(59.5)$ & \\
\hline \multirow[t]{4}{*}{$\begin{array}{l}\text { Employment status: } \mathrm{n} \\
(\%)\end{array}$} & & & & $<0.001$ \\
\hline & Full-time employed & $797(54.7)$ & $661(45.3)$ & \\
\hline & Retired & $210(42.1)$ & $289(57.9)$ & \\
\hline & Other & $530(47.4)$ & $588(52.6)$ & \\
\hline \multirow[t]{5}{*}{ Smoking: n (\%) } & & & & $<.001$ \\
\hline & Yes, daily & $328(45.8)$ & $388(54.2)$ & \\
\hline & Yes, sometimes & $100(39.8)$ & $151(60.2)$ & \\
\hline & No, not anymore & $402(47.7)$ & $441(52.3)$ & \\
\hline & Never smoker & $707(55.9)$ & $558(44.1)$ & \\
\hline \multirow[t]{6}{*}{ Sports activities: n (\%) } & & & & $<0.01$ \\
\hline & No sports activity & $409(49.0)$ & $425(51.0)$ & \\
\hline & Less than one hour a week & $283(45.0)$ & $346(55.0)$ & \\
\hline & Regularly, 1-2 h a week & $356(49.9)$ & $358(50.1)$ & \\
\hline & Regularly, 2-4 h a week & $244(51.6)$ & $229(48.4)$ & \\
\hline & $\begin{array}{c}\text { Regularly, more than } 4 \mathrm{~h} \text { a } \\
\text { week }\end{array}$ & $245(57.6)$ & $180(42.4)$ & \\
\hline Alcohol intake: n (\%) & & & & 0.18 \\
\hline & Daily & $84(45.2)$ & $102(54.8)$ & \\
\hline & Several times per week & $272(48.2)$ & $292(51.8)$ & \\
\hline
\end{tabular}


Table 1. Cont.

\begin{tabular}{|c|c|c|c|c|}
\hline Variables & & $\begin{array}{c}\text { Oral Health-Related } \\
\text { Quality of Life: Scores of } \\
\text { Zero } \\
(\mathbf{N}=1537)\end{array}$ & $\begin{array}{c}\text { Oral Health-Related } \\
\text { Quality of Life: Scores of } \\
\text { One or Higher } \\
(\mathbf{N}=\mathbf{1 5 3 8})\end{array}$ & \\
\hline & Once a week & $271(54.7)$ & $224(45.3)$ & \\
\hline & 1-3 times per month & $271(50.9)$ & $261(49.1)$ & \\
\hline & Less often & $348(48.7)$ & $367(51.3)$ & \\
\hline & Never & $291(49.9)$ & $292(50.1)$ & \\
\hline \multirow[t]{3}{*}{$\begin{array}{l}\text { Vaccinated against } \\
\text { COVID-19: } \mathrm{n}(\%)\end{array}$} & & & & 0.96 \\
\hline & No & $297(50.1)$ & $296(49.9)$ & \\
\hline & Yes & $1240(50.0)$ & $1242(50.0)$ & \\
\hline \multirow[t]{3}{*}{ Chronic diseases: n (\%) } & & & & $<0.001$ \\
\hline & $\begin{array}{c}\text { Absence of chronic } \\
\text { diseases }\end{array}$ & $991(56.1)$ & $774(43.9)$ & \\
\hline & $\begin{array}{l}\text { Presence of at least one } \\
\text { chronic disease }\end{array}$ & $546(41.7)$ & $764(58.3)$ & \\
\hline $\begin{array}{l}\text { Self-rated health }(1=\text { very } \\
\text { bad to } 5=\text { very good }) \text { : mean } \\
\text { (standard deviation) }\end{array}$ & & $3.8(0.8)$ & $3.4(0.9)$ & $<0.001$ \\
\hline Denture usage: n (\%) & & & & $<0.001$ \\
\hline & Without dentures & $1379(53.4)$ & $1203(46.6)$ & \\
\hline & With removable dentures & $122(32.0)$ & $259(68.0)$ & \\
\hline & With complete dentures & $36(32.1)$ & $76(67.9)$ & \\
\hline $\begin{array}{l}\text { Loneliness (from } 1 \text { to } 4 \text {, with } \\
\text { higher values indicating } \\
\text { higher loneliness levels): } \\
\text { mean (standard deviation) }\end{array}$ & & $2.1(0.6)$ & $2.4(0.6)$ & $<0.001$ \\
\hline $\begin{array}{l}\text { Perceived social isolation } \\
\text { (from } 1 \text { to } 4 \text {, with higher } \\
\text { values indicating higher } \\
\text { perceived social isolation } \\
\text { levels): mean (standard } \\
\text { deviation) }\end{array}$ & & $1.7(0.8)$ & $2.1(0.8)$ & $<0.001$ \\
\hline $\begin{array}{c}\text { Objective social isolation } \\
\text { (from } 0 \text { to 30, with higher } \\
\text { values indicating higher } \\
\text { objective social isolation } \\
\text { levels): mean (standard } \\
\text { deviation) }\end{array}$ & & $15.8(6.3)$ & $16.7(5.9)$ & $<0.001$ \\
\hline
\end{tabular}

Notes: Independent $t$-tests or $\mathrm{Chi}^{2}$-tests were conducted, as appropriate.

\subsection{Bivariate Analysis}

It is worth noting that all outcomes are somewhat correlated (loneliness and perceived social isolation: $\mathrm{r}=0.56, p<0.001$; loneliness and objective social isolation: $\mathrm{r}=0.44$, $p<0.001$; perceived social isolation and objective social isolation: $\mathrm{r}=0.27, p<0.001$ ). Additionally, oral health-related quality of life was associated with loneliness $(\mathrm{r}=0.23$, $p<0.001)$, perceived social isolation $(\mathrm{r}=0.30, p<0.001)$ and objective social isolation $(\mathrm{r}=0.08, p<0.001)$. Please also see Table 2 . 
Table 2. Pearson correlations between key variables (total sample).

\begin{tabular}{ccccc}
\hline & $\mathbf{1}$ & $\mathbf{2}$ & $\mathbf{3}$ & $\mathbf{4}$ \\
\hline 1: Loneliness & 1 & & & \\
\hline 2: Perceived social isolation & $0.56^{* * *}$ & 1 & & \\
\hline 3: Objective social isolation & $0.44^{* * *}$ & $0.27^{* * *}$ & 1 & \\
\hline 4: Oral health-related quality of life & $0.23^{* * *}$ & $0.30^{* * *}$ & $0.08^{* * *}$ & 1 \\
\hline Observations & 3075 & 3075 & 3075 & 3075 \\
\hline Notes: Pearson correlations are displayed. ${ }^{* * *} p<0.001$ & & &
\end{tabular}

Stratified by denture usage (1. without dentures; 2 . with removable dentures; 3 . with complete dentures), pairwise correlations were as follows: oral health-related quality of life was associated with loneliness $(1: \mathrm{r}=0.24, p<0.001 ; 2: \mathrm{r}=0.20, p<0.001 ; 3: \mathrm{r}=0.28$, $p<0.01)$, perceived social isolation $(1: \mathrm{r}=0.29, p<0.001 ; 2: \mathrm{r}=0.40, p<0.001 ; 3: \mathrm{r}=0.38$, $p<0.001)$ and objective social isolation $(1: \mathrm{r}=0.08, p<0.001 ; 2: \mathrm{r}=-0.03, p=0.58 ; 3: \mathrm{r}=0.08$, $p=0.42$ ). Thus, among individuals with complete dentures, oral health-related quality of life was only associated with loneliness. Please also see Table 3.

Table 3. Pearson correlations between key variables (stratified by denture usage).

\begin{tabular}{|c|c|c|c|c|c|c|c|c|c|c|c|c|}
\hline & \multicolumn{4}{|c|}{ Without Dentures } & \multicolumn{4}{|c|}{ With Removable Dentures } & \multicolumn{4}{|c|}{ With Complete Dentures } \\
\hline & 1 & 2 & 3 & 4 & 1 & 2 & 3 & 4 & 1 & 2 & 3 & 4 \\
\hline 1: Loneliness & 1 & & & & 1 & & & & 1 & & & \\
\hline $\begin{array}{c}\text { 2: Perceived } \\
\text { social isolation }\end{array}$ & $0.55^{* * *}$ & 1 & & & $0.56^{* * *}$ & 1 & & & $0.63^{* * *}$ & 1 & & \\
\hline $\begin{array}{c}\text { 3: Objective } \\
\text { social isolation }\end{array}$ & $0.43^{* * *}$ & $0.28^{* * *}$ & 1 & & $0.43^{* * *}$ & $0.23^{* * *}$ & 1 & & $0.54^{* * *}$ & $0.23 *$ & 1 & \\
\hline $\begin{array}{c}\text { 4: Oral } \\
\text { health-related } \\
\text { quality of life }\end{array}$ & $0.24^{* * *}$ & $0.29 * * *$ & $0.08^{* * *}$ & 1 & $0.20^{* * *}$ & $0.40^{* * *}$ & -0.03 & 1 & $0.28^{* *}$ & $0.38^{* * *}$ & 0.08 & 1 \\
\hline Observations & \multicolumn{4}{|c|}{2582} & \multicolumn{4}{|c|}{381} & \multicolumn{4}{|c|}{112} \\
\hline
\end{tabular}

Notes: Pearson correlations are displayed. ${ }^{* * *} p<0.001,{ }^{* *} p<0.01,{ }^{*} p<0.05$.

\subsection{Regression Analysis}

Findings of multiple linear regressions for the total sample are shown in Table 4.

Table 4. Determinants of loneliness, perceived social isolation and objective social isolation. Results of multiple linear regressions.

\begin{tabular}{cccc}
\hline Independent Variables & Loneliness & Perceived Social Isolation & Objective Social Isolation \\
\hline Oral health-related quality of life & B (robust standard errors) & B (robust standard errors) & B (robust standard errors) \\
& $0.03^{* * *}$ & $0.06^{* * *}$ & $0.07^{*}$ \\
Potential confounders & $(0.00)$ & $(0.00)$ & $(0.03)$ \\
Observations & $\checkmark$ & $\checkmark$ & $\checkmark$ \\
Adjusted R & 3075 & 0.24 & 3075 \\
$\mathrm{R}^{2}$ & 0.17 & 0.24 & 0.16 \\
F-value $(p$-value) & 0.18 & $37.39(p<0.001)$ & 0.17 \\
\hline
\end{tabular}

Notes: Unstandardized beta-coefficients (B) are displayed; robust standard errors in parentheses; ${ }^{* * *} p<0.001,{ }^{*} p<0.05$; Potential confounders $(\checkmark)$ include sex, age, marital status, education, presence of children in the same household, smoking status, alcohol intake, sports activities, vaccinated against COVID-19, presence of chronic diseases and self-rated health. 
Regressions showed that low oral health-related quality of life is associated with higher loneliness $(B=0.03, p<0.001)$, higher perceived social isolation $(B=0.06, p<0.001)$ and higher objective social isolation $(B=0.07, p<0.05)$.

When the OHIP-G5 was dichotomized (first category: score of zero; second category: scores of one or higher), scores of one or higher were associated with higher loneliness $(B=0.20, p<0.001)$, higher perceived social isolation $(B=0.28, p<0.001)$ and higher objective social isolation $(B=0.46, p<0.05)$ (please see Supplementary Table $\mathrm{S} 1$ ).

Stratified by denture usage, multiple linear regressions are shown in Table 5.

Table 5. Determinants of loneliness, perceived social isolation and objective social isolation (stratified by denture usage: 1 . without dentures, 2. With removable dentures; 3. with complete dentures). Results of multiple linear regressions.

\begin{tabular}{|c|c|c|c|c|c|c|c|c|c|}
\hline \multirow[b]{2}{*}{$\begin{array}{l}\text { Independent } \\
\text { Variables }\end{array}$} & \multicolumn{3}{|c|}{ Without Dentures } & \multicolumn{3}{|c|}{ With Removable Dentures } & \multicolumn{3}{|c|}{ With Complete Dentures } \\
\hline & Loneliness & $\begin{array}{l}\text { Perceived } \\
\text { Social } \\
\text { Isolation }\end{array}$ & $\begin{array}{c}\text { Objective } \\
\text { Social } \\
\text { Isolation }\end{array}$ & Loneliness & $\begin{array}{c}\text { Perceived } \\
\text { Social } \\
\text { Isolation }\end{array}$ & $\begin{array}{c}\text { Objective } \\
\text { Social } \\
\text { Isolation }\end{array}$ & Loneliness & $\begin{array}{c}\text { Perceived } \\
\text { Social } \\
\text { Isolation }\end{array}$ & $\begin{array}{c}\text { Objective } \\
\text { Social } \\
\text { Isolation }\end{array}$ \\
\hline & $\begin{array}{c}\text { B } \\
\text { (robust } \\
\text { standard } \\
\text { errors) }\end{array}$ & $\begin{array}{c}\text { B } \\
\text { (robust } \\
\text { standard } \\
\text { errors) }\end{array}$ & $\begin{array}{c}\text { B } \\
\text { (robust } \\
\text { standard } \\
\text { errors) }\end{array}$ & $\begin{array}{c}\text { B } \\
\text { (robust } \\
\text { standard } \\
\text { errors) }\end{array}$ & $\begin{array}{c}\text { B } \\
\text { (robust } \\
\text { standard } \\
\text { errors) }\end{array}$ & $\begin{array}{c}\text { B } \\
\text { (robust } \\
\text { standard } \\
\text { errors) }\end{array}$ & $\begin{array}{c}\text { B } \\
\text { (robust } \\
\text { standard } \\
\text { errors) }\end{array}$ & $\begin{array}{c}\text { B } \\
\text { (robust } \\
\text { standard } \\
\text { errors) }\end{array}$ & $\begin{array}{c}\text { B } \\
\text { (robust } \\
\text { standard } \\
\text { errors) }\end{array}$ \\
\hline \multirow[t]{2}{*}{$\begin{array}{l}\text { Oral health- } \\
\text { related } \\
\text { quality of } \\
\text { life }\end{array}$} & $0.04 * * *$ & $0.06^{* * *}$ & $0.08 *$ & 0.01 & $0.05^{* * *}$ & -0.03 & $0.04 *$ & $0.06^{* *}$ & 0.18 \\
\hline & $(0.00)$ & $(0.01)$ & $(0.04)$ & $(0.01)$ & $(0.01)$ & $(0.08)$ & $(0.02)$ & $(0.02)$ & $(0.17)$ \\
\hline $\begin{array}{l}\text { Potential } \\
\text { con- } \\
\text { founders }\end{array}$ & $\checkmark$ & $\checkmark$ & $\checkmark$ & $\checkmark$ & $\checkmark$ & $\checkmark$ & $\checkmark$ & $\checkmark$ & $\checkmark$ \\
\hline Observations & 2582 & 2582 & 2582 & 381 & 381 & 381 & 112 & 112 & 112 \\
\hline $\begin{array}{c}\text { Adjusted } \\
\mathrm{R}^{2}\end{array}$ & 0.17 & 0.23 & 0.16 & 0.14 & 0.31 & 0.08 & 0.24 & 0.38 & 0.14 \\
\hline $\mathrm{R}^{2}$ & 0.18 & 0.24 & 0.17 & 0.20 & 0.36 & 0.15 & 0.43 & 0.54 & 0.36 \\
\hline $\begin{array}{l}\text { F-value } \\
\text { ( } p \text {-value })\end{array}$ & $\begin{array}{c}19.39 \\
(p<0.001)\end{array}$ & $\begin{array}{c}29.20 \\
(p<0.001)\end{array}$ & $\begin{array}{c}18.51 \\
(p<0.001)\end{array}$ & $\begin{array}{c}13.19 \\
(p<0.001)\end{array}$ & $\begin{array}{c}58.95 \\
(p<0.001)\end{array}$ & $\begin{array}{c}28.99 \\
(p<0.001)\end{array}$ & $\begin{array}{c}16.09 \\
(p<0.001)\end{array}$ & $\begin{array}{c}44.41 \\
(p<0.001)\end{array}$ & $\begin{array}{c}22.32 \\
(p<0.001)\end{array}$ \\
\hline
\end{tabular}

Notes: Unstandardized beta-coefficients are displayed; Robust standard errors in parentheses; ${ }^{* * *} p<0.001,{ }^{* *} p<0.01,{ }^{*} p<0.05$; Potential confounders $(\checkmark)$ include sex, age, marital status, education, presence of children in the same household, smoking status, alcohol intake, sports activities, vaccinated against COVID-19, presence of chronic diseases and self-rated health.

Among individuals without dentures, oral health-related quality of life was associated with higher loneliness $(B=0.04, p<0.001)$, higher perceived social isolation $(B=0.06$, $p<0.001)$ and higher objective social isolation $(B=0.08, p<0.05)$.

Among individuals with removable dentures, oral health-related quality of life was associated with higher perceived social isolation $(B=0.05, p<0.001)$, but not with loneliness and objective social isolation.

Among individuals with complete dentures, oral health-related quality of life was associated with higher loneliness $(\mathrm{B}=0.04, p<0.05)$ and higher perceived social isolation $(\mathrm{B}=0.06, p<0.01)$, but not with objective social isolation.

However, when examining the moderating effect of denture usage on the association between oral health-related quality of life and the outcomes (by including respective interaction terms), nearly all interaction terms did not achieve statistical significance (except for the interaction term between 'with removable dentures' and oral-health related quality of life, with loneliness as the outcome measure: $\mathrm{B}=-0.02, p=0.02$ ) (please see Supplementary Table S2). 


\section{Discussion}

\subsection{Main Findings}

Based on data from a large, nationally representative sample, the aim of our study was to clarify the association between oral health-related quality of life and loneliness, perceived social isolation as well as objective social isolation. Pairwise correlations between oral health-related quality of life and all outcomes were small to medium (from 0.1 to 0.3). Regressions showed that low oral health-related quality of life is associated with higher loneliness, higher perceived social isolation and higher objective social isolation. Furthermore, associations were shown stratified by denture usage. Our study extends previous knowledge regarding the association between oral health-related quality of life and social needs mainly based on small samples in specific regions (and among children or individuals in late life).

\subsection{Previous Research and Possible Explanations}

Our study demonstrated an association between oral health-related quality of life and loneliness, perceived social isolation as well as objective social isolation.

With regard to the association between oral health-related quality of life and loneliness, our study is mainly in accordance with previous studies (typically based on small samples), showing an association between objective oral health $[9,12]$ as well as oral-health related quality of life and loneliness [14].

While two studies exclusively focusing on children and adolescents did not find an association between objective oral health or oral health-related quality of life and objective social isolation $[7,15]$, two other studies found an association between lower objective oral health and higher objective social isolation among older adults $[8,11]$. Furthermore, one study showed an association between lower objective oral health and higher objective social isolation among children [13]. Our study adds to this current knowledge based on samples from children or individuals in late life. Furthermore, we added knowledge regarding the association between oral health-related quality of life and perceived social isolation.

Several mechanisms may explain the association between oral health-related quality of life and the outcomes used in our study. For example, low oral health is associated with low mental health [30]. This in turn is associated with higher loneliness as well as higher social isolation [31,32]. Moreover, studies have demonstrated that oral health is associated with the risk of homebound status, which in turn can lead to feelings of loneliness or isolation [33]. Similarly, low oral health-related quality of life may increase factors such as shame, embarrassment and perceived stigma of being poor or may contribute to low self-esteem [34,35]—which in turn may lead to loneliness and isolation [36]. Additionally, factors such as social status, social class or mental health may be important for the association between oral health-related quality of life and the outcomes used in our study.

Our study also showed regressions stratified by denture usage. However, the sample size differed markedly between the groups. Thus, non-significant associations (particularly in the second and third group) may be particularly explained by a lack of statistical power.

\subsection{Strengths and Limitations}

For our study, data were used from a nationally-representative sample. Furthermore, as the first study in this area, we concentrated on the association between oral healthrelated quality of life and (i) loneliness, (ii) perceived social isolation and (iii) objective social isolation to get a more comprehensive picture. Widely used and well-established tools were used to quantify oral health-related quality of life and the social needs. It was adjusted for several potential confounders in regression analysis. Due to the cross-sectional design, it is difficult to draw causal conclusions. It also appears to be plausible that oral health-related quality of life contributes to loneliness or social isolation. Therefore, future longitudinal studies are needed in this area. 


\section{Conclusions}

Our study showed the relevance of low oral health-related quality of life for loneliness and perceived social isolation as well as objective social isolation. Future studies should elucidate the underlying mechanisms. Moreover, future research is required to clarify the association between oral health-related quality of life and loneliness, perceived social isolation as well as objective social isolation in other countries.

Supplementary Materials: The following are available online at https:/ / www.mdpi.com/article/ 10.3390/ijerph182412886/s1, Table S1: Determinants of loneliness, perceived social isolation and objective social isolation (with dichotomized oral health-related quality of life). Results of multiple linear regressions, Table S2: Determinants of loneliness, perceived social isolation and objective social isolation (with interaction terms: denture usage $x$ oral health-related quality of life). Results of multiple linear regressions.

Author Contributions: Conceptualization, A.H. and H.-H.K. Methodology, A.H.; Data curation, A.H.; Visualization, A.H. and H.-H.K.; Writing-original draft preparation, A.H.; Writing-review and editing, A.H. and H.-H.K. Formal analysis, A.H.; Supervision: H.-H.K. All authors have read and agreed to the published version of the manuscript.

Funding: This research received no external funding.

Institutional Review Board Statement: The study was conducted according to the guidelines of the Declaration of Helsinki, and approved by the Local Psychological Ethics Committee of the Center for Psychosocial Medicine of the University Medical Center Hamburg-Eppendorf (number: LPEK-0356).

Informed Consent Statement: Informed consent was obtained from all subjects involved in the study.

Data Availability Statement: The datasets used and analyzed during the current study are available from the corresponding author on reasonable request from all interested researchers.

Conflicts of Interest: The authors declare no conflict of interest.

\section{References}

1. Holt-Lunstad, J.; Smith, T.B.; Baker, M.; Harris, T.; Stephenson, D. Loneliness and social isolation as risk factors for mortality: A meta-analytic review. Perspect. Psychol. Sci. 2015, 10, 227-237. [CrossRef]

2. Luanaigh, C.Ó.; Lawlor, B.A. Loneliness and the health of older people. Int. J. Geriatr. Psychiatry A J. Psychiatry Late Life Allied Sci. 2008, 23, 1213-1221. [CrossRef] [PubMed]

3. Hajek, A.; Kretzler, B.; König, H.-H. The Association Between Obesity and Social Isolation as Well as Loneliness in the Adult Population: A Systematic Review. Diabetes Metab. Syndr. Obes. Targets Ther. 2021, 14, 2765. [CrossRef]

4. Ejiri, M.; Kawai, H.; Ishii, K.; Oka, K.; Obuchi, S. Predictors of older adults' objectively measured social isolation: A systematic review of observational studies. Arch. Gerontol. Geriatr. 2021, 94, 104357. [CrossRef]

5. Hajek, A.; Kretzler, B.; König, H.-H. Multimorbidity, loneliness, and social isolation. A systematic review. Int. J. Environ. Res. Public Health 2020, 17, 8688. [CrossRef] [PubMed]

6. Dahlberg, L.; McKee, K.J.; Frank, A.; Naseer, M. A systematic review of longitudinal risk factors for loneliness in older adults. Aging Ment. Health 2021, 1-25. [CrossRef] [PubMed]

7. Delgado-Angulo, E.K.; Hobdell, M.H.; Bernabé, E. Poverty, social exclusion and dental caries of 12-year-old children: A cross-sectional study in Lima, Peru. BMC Oral Health 2009, 9, 16. [CrossRef]

8. Koyama, S.; Saito, M.; Cable, N.; Ikeda, T.; Tsuji, T.; Noguchi, T.; Abbas, H.; Miyashiro, I.; Osaka, K.; Kondo, K.; et al. Examining the associations between oral health and social isolation: A cross-national comparative study between Japan and England. Soc. Sci. Med. 2021, 277, 113895. [CrossRef]

9. Lundgren, M.; Osterberg, T.; Emilson, G.; Steen, B. Oral complaints and utilization of dental services in relation to general health factors in a 88-year-old Swedish population. Gerodontology 1995, 12, 81-88. [CrossRef] [PubMed]

10. Monteiro da Silva, A.M.; Oakley, D.A.; Newman, H.N.; Nohl, F.S.; Lloyd, H.M. Psychosocial factors and adult onset rapidly progressive periodontitis. J. Clin. Periodontol. 1996, 23, 789-794. [CrossRef] [PubMed]

11. Olofsson, H.; Ulander, E.L.; Gustafson, Y.; Hörnsten, C. Association between socioeconomic and health factors and edentulism in people aged 65 and older-a population-based survey. Scand. J. Public Health 2018, 46, 690-698. [CrossRef]

12. Singh, A.; Purohit, B.M.; Taneja, S. Loneliness and disability as predictors of oral diseases among 2 groups of older adults in central India. J. Am. Dent Assoc. 2020, 151, 427-437. [CrossRef] [PubMed]

13. Vazquez Fde, L.; Cortellazzi, K.L.; Kaieda, A.K.; Bulgareli, J.V.; Mialhe, F.L.; Ambrosano, G.M.; da Silva Tagliaferro, E.P.; Guerra, L.M.; de Castro Meneghim, M.; Pereira, A.C. Individual and contextual factors related to dental caries in underprivileged Brazilian adolescents. BMC Oral Health 2015, 15, 6. [CrossRef] [PubMed] 
14. Rouxel, P.; Heilmann, A.; Demakakos, P.; Aida, J.; Tsakos, G.; Watt, R.G. Oral health-related quality of life and loneliness among older adults. Eur. J. Ageing 2017, 14, 101-109. [CrossRef]

15. Vazquez Fde, L.; Cortellazzi, K.L.; Kaieda, A.K.; Guerra, L.M.; Ambrosano, G.M.; Tagliaferro, E.P.; Mialhe, F.L.; Meneghim Mde, C.; Pereira, A.C. Quality of life and socio-dental impact among underprivileged Brazilian adolescents. Qual. Life Res. 2015, 24, 661-669. [CrossRef] [PubMed]

16. Münnich, R.; Gabler, S. 2012: Stichprobenoptimierung und Schätzung in Zensus 2011; Statistisches Bundesamt: Wiesbaden, Germany, 2012; Volume 21.

17. Wenger, G.C.; Davies, R.; Shahtahmasebi, S.; Scott, A. Social isolation and loneliness in old age: Review and model refinement. Ageing Soc. 1996, 16, 333-358. [CrossRef]

18. Bude, H.; Lantermann, E.-D. Soziale exklusion und exklusionsempfinden. KZfSS Kölner Z. Für Soziologie Und Soz. 2006, 58, 233-252. [CrossRef]

19. Dahlberg, L. Loneliness during the COVID-19 pandemic. Aging Ment. Health 2021, 25, 1-4. [CrossRef]

20. Hajek, A.; König, H.-H. Do Loneliness and Perceived Social Isolation Reduce Expected Longevity and Increase the Frequency of Dealing with Death and Dying? Longitudinal Findings based on a Nationally Representative Sample. J. Am. Med Dir. Assoc. 2021, 22, 1720-1725.e5. [CrossRef]

21. Gierveld, J.D.J.; Tilburg, T.V. A 6-item scale for overall, emotional, and social loneliness: Confirmatory tests on survey data. Res. Aging 2006, 28, 582-598. [CrossRef]

22. Chiao, C.; Chen, Y.-H.; Yi, C.-C. Loneliness in young adulthood: Its intersecting forms and its association with psychological well-being and family characteristics in Northern Taiwan. PLoS ONE 2019, 14, e0217777. [CrossRef]

23. van den Broek, T.; Grundy, E. Loneliness among Polish migrants in the Netherlands: The impact of presence and location of partners and offspring. Demogr. Res. 2017, 37, 727-742. [CrossRef]

24. Victor, C.R.; Rippon, I.; Nelis, S.M.; Martyr, A.; Litherland, R.; Pickett, J.; Hart, N.; Henley, J.; Matthews, F.; Clare, L. Prevalence and determinants of loneliness in people living with dementia: Findings from the IDEAL programme. Int. J. Geriatr. Psychiatry 2020, 35, 1-8. [CrossRef] [PubMed]

25. Gierveld, J.D.J.; Van Tilburg, T. The De Jong Gierveld short scales for emotional and social loneliness: Tested on data from 7 countries in the UN generations and gender surveys. Eur. J. Ageing 2010, 7, 121-130. [CrossRef] [PubMed]

26. Lubben, J.; Blozik, E.; Gillmann, G.; Iliffe, S.; von Renteln Kruse, W.; Beck, J.C.; Stuck, A.E. Performance of an abbreviated version of the Lubben Social Network Scale among three European community-dwelling older adult populations. Gerontology 2006, 46, 503-513. [CrossRef]

27. John, M.T.; Miglioretti, D.L.; LeResche, L.; Koepsell, T.D.; Hujoel, P.; Micheelis, W. German short forms of the oral health impact profile. Community Dent. Oral Epidemiol. 2006, 34, 277-288. [CrossRef]

28. John, M.T.; Micheelis, W.; Biffar, R. Orginalarbeiten-Einflussfaktoren mundgesundheitsbezogener Lebensqualitat-Validierung einer deutschen Kurzversion des Oral Health Impact Profile (OHIP-G14). Dtsch. Zahnarztl. Z. 2004, 59, 328-333.

29. John, M.T.; Koepsell, T.D.; Hujoel, P.; Miglioretti, D.L.; LeResche, L.; Micheelis, W. Demographic factors, denture status and oral health-related quality of life. Community Dent. Oral Epidemiol. 2004, 32, 125-132. [CrossRef]

30. Cademartori, M.G.; Gastal, M.T.; Nascimento, G.G.; Demarco, F.F.; Corrêa, M.B. Is depression associated with oral health outcomes in adults and elders? A systematic review and meta-analysis. Clin. Oral Investig. 2018, 22, 2685-2702. [CrossRef] [PubMed]

31. Cacioppo, J.T.; Hughes, M.E.; Waite, L.J.; Hawkley, L.C.; Thisted, R.A. Loneliness as a specific risk factor for depressive symptoms: Cross-sectional and longitudinal analyses. Psychol. Aging 2006, 21, 140-151. [CrossRef]

32. Coyle, C.E.; Dugan, E. Social isolation, loneliness and health among older adults. J. Aging Health 2012, 24, 1346-1363. [CrossRef] [PubMed]

33. Koyama, S.; Aida, J.; Kondo, K.; Yamamoto, T.; Saito, M.; Ohtsuka, R.; Nakade, M.; Osaka, K. Does poor dental health predict becoming homebound among older Japanese? BMC Oral Health 2016, 16, 1-9. [CrossRef] [PubMed]

34. Benyamini, Y.; Leventhal, H.; Leventhal, E.A. Self-rated oral health as an independent predictor of self-rated general health, self-esteem and life satisfaction. Soc. Sci. Med. 2004, 59, 1109-1116. [CrossRef] [PubMed]

35. Locker, D. Self-esteem and socioeconomic disparities in self-perceived oral health. J. Public Health Dent. 2009, 69, 1-8. [CrossRef] [PubMed]

36. Heatherton, T.F.; Wyland, C.L. Assessing self-esteem. In Positive Psychological Assessment: A Handbook of Models and Measures; Lopez, S.J., Snyder, C.R., Eds.; American Psychological Association: Washington, DC, USA, 2003. 